\title{
Large serological survey showing cocirculation of Ebola and Marburg viruses in Gabonese bat populations, and a high seroprevalence of both viruses in Rousettus aegyptiacus
}

\author{
Xavier Pourrut*1,2, Marc Souris ${ }^{1,3}$, Jonathan S Towner ${ }^{4}$, Pierre E Rollin ${ }^{4}$, \\ Stuart T Nichol ${ }^{4}$, Jean-Paul Gonzalez ${ }^{2}$ and Eric Leroy ${ }^{1,2}$
}

Address: ${ }^{1}$ Institut de Recherche pour le Développement, UR 178, Marseille, France, ${ }^{2}$ Centre International de Recherches Médicales, BP 769 , Franceville, Gabon, ${ }^{3}$ Institut de Recherche pour le Développement, UR 178, Mahidol University at Salaya, 25/25 Phutthamonthon 4 , Nakhonpathon 73170, Thaïland and ${ }^{4}$ Special Pathogens Branch, Centers for Disease Control and Prevention, Atlanta, Georgia, USA

Email: Xavier Pourrut* - xavier.pourrut@ird.fr; Marc Souris - fnmsr@diamond.mahidol.ac.th; Jonathan S Towner - jit8@cdc.gov; Pierre E Rollin - pyr3@cdc.gov; Stuart T Nichol - stn1@CDC.GOV; Jean-Paul Gonzalez - Jean-paul.gonzalez@ird.fr; Eric Leroy - eric.leroy@ird.fr

* Corresponding author

Published: 28 September 2009

BMC Infectious Diseases 2009, 9:159 doi:10.1186/147/-2334-9-159

This article is available from: http://www.biomedcentral.com/l47/-2334/9/159

(c) 2009 Pourrut et al; licensee BioMed Central Ltd.

This is an Open Access article distributed under the terms of the Creative Commons Attribution License (http://creativecommons.org/licenses/by/2.0), which permits unrestricted use, distribution, and reproduction in any medium, provided the original work is properly cited.
Received: 3 March 2009

Accepted: 28 September 2009

\begin{abstract}
Background: Ebola and Marburg viruses cause highly lethal hemorrhagic fevers in humans. Recently, bats of multiple species have been identified as possible natural hosts of Zaire ebolavirus (ZEBOV) in Gabon and Republic of Congo, and also of marburgvirus (MARV) in Gabon and Democratic Republic of Congo.
\end{abstract}

Methods: We tested 2147 bats belonging to at least nine species sampled between 2003 and 2008 in three regions of Gabon and in the Ebola epidemic region of north Congo for IgG antibodies specific for ZEBOV and MARV.

Results: Overall, IgG antibodies to ZEBOV and MARV were found in $4 \%$ and $1 \%$ of bats, respectively. ZEBOV-specific antibodies were found in six bat species (Epomops franqueti, Hypsignathus monstrosus, Myonycteris torquata, Micropteropus pusillus, Mops condylurus and Rousettus aegyptiacus), while MARV-specific antibodies were only found in Rousettus aegyptiacus and Hypsignathus monstrosus. The prevalence of MARV-specific IgG was significantly higher in $R$. aegyptiacus members captured inside caves than elsewhere. No significant difference in prevalence was found according to age or gender. A higher prevalence of ZEBOV-specific IgG was found in pregnant females than in non pregnant females.

Conclusion: These findings confirm that ZEBOV and MARV co-circulate in Gabon, the only country where bats infected by each virus have been found. IgG antibodies to both viruses were detected only in Rousettus aegyptiacus, suggesting that this bat species may be involved in the natural cycle of both Marburg and Ebola viruses. The presence of MARV in Gabon indicates a potential risk for a first human outbreak. Disease surveillance should be enhanced in areas near caves. 


\section{Background}

Filoviridae, a family of single-stranded non segmented RNA viruses, comprises two genera, marburgvirus (MARV) and ebolavirus (EBOV), the latter comprising Zaire ebolavirus (ZEBOV), Sudan ebolavirus (SEBOV), Cote d'Ivoire ebolavirus (CIEBOV), Reston ebolavirus (REBOV) and Bundibungyo ebolavirus (BEBOV) [1,2]. Filoviruses cause hemorrhagic fever outbreaks in humans, with high case fatality rates (except for REBOV). Seventeen outbreaks and two isolated cases of human EBOV infection have been reported, in Democratic Republic of Congo (DRC) (1976, 1977, 1995, 2007, 2008), Sudan (1976, 1979, 2004), Gabon (1994, 1996, 1997, 2001), Republic of Congo (RC) $(2001,2003,2005)$, Ivory Coast (1995) and Uganda $(2000,2007)[2-4]$. Three outbreaks and four isolated cases of human MARV infection have been reported, in Germany and the former Yugoslavia (1967), Zimbabwe (1975), Kenya (1980, 1987), DRC (1998-1999), Angola (2005) and Uganda (2007) [5]. Consistent with this epidemiological pattern, ecologic niche modeling of outbreaks and isolated cases has suggested that Ebola hemorrhagic fever occurs in the rain forests of central and western Africa, and Marburg in more open areas of central and western Africa [6,7]. In Gabon and RC, most human ZEBOV outbreaks have occurred through contact with infected animal carcasses, especially great apes and duikers [8]. Bats were recently identified as potential reservoir species of these filoviruses $[3,9,10]$. During the ZEBOV outbreaks that occurred between 2001 and 2003 in Gabon and RC, numerous animals were captured in the search for reservoir species. Of the 1024 small mammals sampled, including 679 bats, ZEBOV-specific antibodies were detected in the serum of $16(8 \%)$ of 192 fruit bats belonging to three species, namely Hypsignathus monstrosus, Epomops franqueti, and Myonycteris torquata; ZEBOV RNA was detected in pooled liver and spleen samples from $13(5 \%)$ of 279 such bats [11]. Sequence analysis indicated that bats of these species might serve as a ZEBOV natural host. A large-scale serological survey of bats belonging to these three species was then conducted, with 1390 specimens captured between 2003 and 2006 in three regions of Gabon and in the epidemic border region with RC. The prevalence of ZEBOV-specific IgG was about $5 \%$ in bats from all four regions, and fell to about $1 \%$ at all four sites a year after the most recent Ebola outbreak, which occurred in 2005 in RC [12]. These results indicate that ZEBOV-specific antibodies are widely distributed in these bats in Gabon and RC, supporting their potential reservoir status. As a Marburg outbreak occurred in 2005 in Angola, $800 \mathrm{~km}$ from the Gabon border, we also tested bats for signs of MARV infection. Of 438 bats collected in Gabon and RC, MARV-specific IgG was detected in the serum of 29 of 242 specimens of Rousettus aegyptiacus, and MARV nucleotide sequences were detected in four members of 283 bats of the same species, suggesting that $R$. aegyptiacus bats may be a MARV reservoir [13]
In the present study, we documented the distribution of ZEBOV- and MARV-specific antibodies in bat communities living in forest areas of Gabon and northern RC. We report detailed serologic results for 2147 bats belonging to at least nine species sampled between 2003 and 2008.

\section{Methods \\ Collection sites and periods}

Samples were collected in the Republic of Congo and Gabon (Figure 1).

In the Republic of Congo, two sites were sampled near Mbomo $\left(0^{\circ} 25^{\prime} \mathrm{O} ; 14^{\circ} 41^{\prime} \mathrm{E}\right)$ and Odzala National Park. The vegetation includes dense semi-deciduous and evergreen forest with open and closed canopies. The climate is equatorial, with two dry seasons (December-February and June-August) and two wet seasons (March-May and September-November). In Gabon, bats were collected in three different regions. The first was situated in HautOgooué province in the south-east of the country (near Franceville $1^{\circ} 37^{\prime} \mathrm{S} ; 13^{\circ} 36^{\prime} \mathrm{E}$ ). Four sites comprising forested areas, savannas and a cave were sampled. The vegetation forms a savanna-forest mosaic. The climate is tropical-transitional, with one clear dry season (JuneAugust) and one long wet season (September-May), with lower rainfall in December and January. The second region was located in Moyen-Ogooué province in west Gabon (near Lambaréné $0^{\circ} 41^{\prime} \mathrm{S} ; 1^{\circ} 1^{\circ} 01^{\prime} \mathrm{E}$ ). Bats were collected from three different biotopes. The climate is tropical-transitional and humid. The forest vegetation is semideciduous with lakes, rivers and swamps. The third region was in Nyanga province, in south-west Gabon (near Tchibanga $2^{\circ} 51^{\prime} \mathrm{S} ; 11^{\circ} 01^{\prime} \mathrm{E}$ ). The vegetation is composed of an evergreen forest with savanna areas and caves. The climate is tropical with a long dry season (May-September) and a long wet season (October-April).

\section{Collection periods}

In RC, bats were collected both during ZEBOV outbreaks in June 2003 and May 2005 and also one year after the last outbreak in May 2006. In Gabon, bats were collected in Haut-Ogooué in February 2005, October 2006 and March 2008, in Moyen Ogooué in April 2005, and in Nyanga in February 2006.

\section{Bat trapping and blood sampling}

Bats were trapped with mist nets. The nets were set in the early evening, and captured animals were removed each morning. The captured bats were transported to a field laboratory specially designed for this study, a few kilometers from the capture sites, where they were euthanized. Weight and body measurements, sexing and species determination were performed as previously described [12]. Blood was collected in EDTA tubes by cardiac puncture and plasma was obtained by centrifugation at $3000 \mathrm{rpm}$ for $10 \mathrm{~min}$, then placed in Cryovials (VWR Prolabo, Fon- 


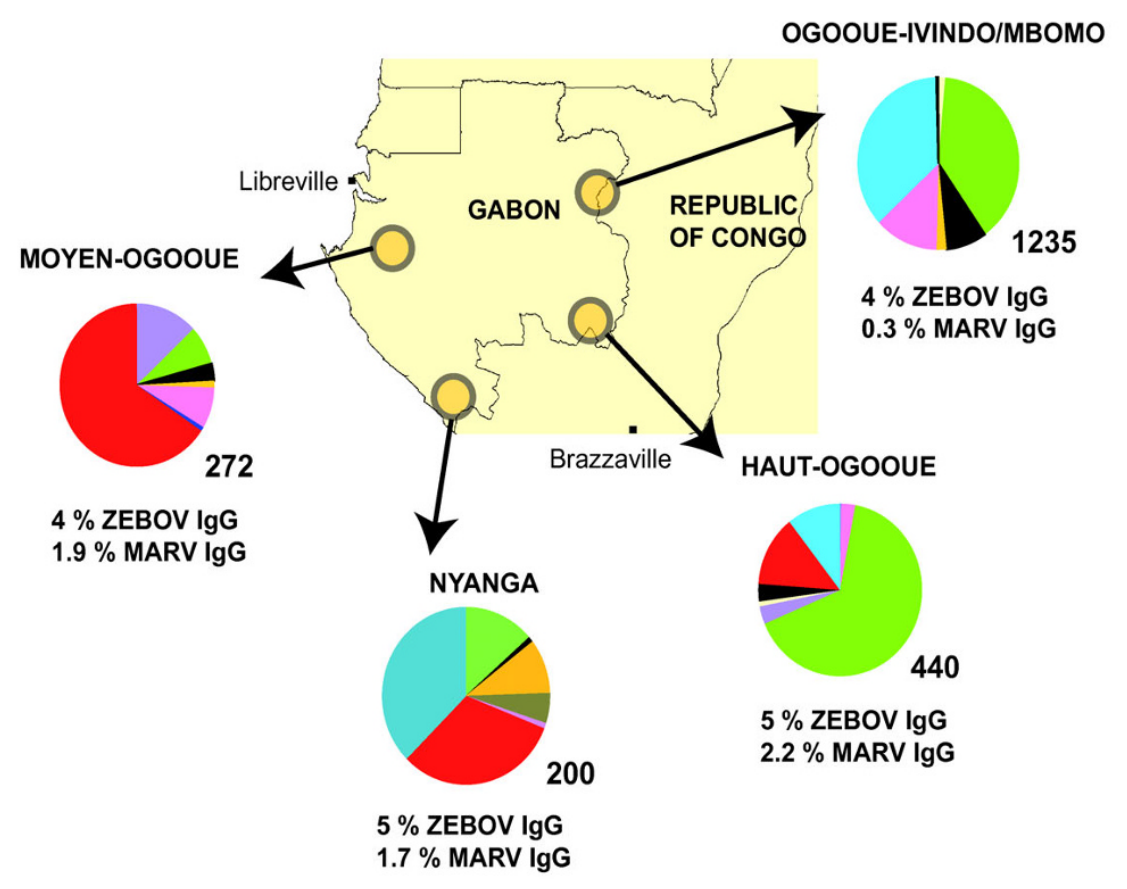

Casinycteris argynnis

Eidolon helvum

Epomops franqueti

Hypsignathus monstrosus

Megaloglossus woermanni

Microchiroptera *

Micropteropus pusillus

Myonycteris torquata

Rousettus aegyptiacus

* Including Mops condylurus and Hipposideros gigas

\section{Figure I}

Map of Gabon and Republic of Congo. Sampling sites and sampled bat species are shown. The circles represent the sampling sites in Gabon and RC. The distribution (percentage) of the different captured bat species is represented by the different colors indicated in the box.

tenay-sous-Bois, France) and stored in liquid nitrogen. Fragments of liver and spleen were obtained by necropsy and immediately frozen. At the end of each capture period the samples were transported to Franceville for analysis at the Centre International de Recherches Médicales de Franceville (CIRMF), Gabon. For ethical aspects, a special authorization was delivered for this study by the Ministry of Health of Gabon (Pr Kombila Kouma Pierre, n ${ }^{\circ} 104 /$ MSPP/SG/DGS, on 17 February 2005).

\section{Serological studies}

Sera were tested with a standard IgG ELISA as previously described [12]. Briefly, ZEBOV antigens diluted 1:1000 in phosphate buffered saline were passively adsorbed overnight at $+4^{\circ} \mathrm{C}$ onto ELISA Maxisorp plates (VWR Prolabo, Fontenay-sous-Bois, France). The protocol was the same for MARV antigens. Control wells were coated with unin- fected cultured Vero cell antigens (mock antigens). The plates were washed three times with PBS-0.1\% Tween 20. One hundred microliters of each serum sample diluted 1:100 in PBS- $0.1 \%$ Tween 20 containing 5\% fat-free milk powder (Becton Dickinson, Temse, Belgium) was added to each well and incubated overnight at $+4^{\circ} \mathrm{C}$. After three washes, $100 \mu \mathrm{l}$ of a 1:1 mixture of peroxidase-labeled protein A ( $1 \mathrm{mg} / \mathrm{ml})$ (Sigma, Taufkirchen, Germany) and peroxidase-labeled protein G $(1 \mathrm{mg} / \mathrm{ml})$ (Sigma, Taufkirchen, Germany) diluted $1: 1000$ in PBS-0.1\% Tween 20 containing 5\% fat-free milk was added to each well. The plates were incubated for one hour at room temperature and washed. TMB substrate (Thermo Electron Corporation, Saint Herblain, France) was used for detection. Optical density (OD) was measured at $450 \mathrm{~nm}$ with a PR 5100 ELISA plate reader (Biorad, Marnes-laCoquette, France). All samples were also tested by using 
HPR-conjugated goat anti-bat IgG ( $1 \mathrm{mg} / \mathrm{ml}$ ) (Bethyl Laboratories, Montgomery, USA) diluted 1:2000 in PBS-0.1\% Tween 20 containing 5\% fat-free milk. Samples from bats with corrected OD values $>0.31$ (see below) were additionally tested at 1:400 and 1:1600 dilutions.

\section{Cut-off determination}

The specific activity of each serum at each dilution (corrected $O D$ ) was calculated by subtracting the non-specific background OD (wells with mock antigen) from the OD of wells with virus antigens. The cut-off value was calculated assuming a negative exponential distribution of positive OD values for negative bats. The parameters of the exponential distribution were derived from the distribution of the collected OD values and a risk of error in order to discard false-positives. With a 0.001 risk of error, the cut-off was 0.31 for both Ebola and Marburg viruses (Figure 2).

\section{Virological studies}

Specific ZEBOV and MARV RNAs were detected in liver and spleen by means of real-time and nested PCR amplification as previously described [12,13]. For each bat, approximately $100 \mathrm{mg}$ of tissue was incubated overnight at $4{ }^{\circ} \mathrm{C}$ in $450 \mu \mathrm{l}$ of cold $2 \times$ cellular lysis buffer (Applied Biosystems International, France) to inactivate viruses. Each tissue was then diluted to $1 \times$ and homogenized for 2 minutes, at 1500 strokes/min using a ball-mill tissue grinder (Geno/grinder 2000, BTC and OPS Diagnostics, Bridgewater, USA). Total RNA was extracted from $\sim 150 \mu \mathrm{l}$ of the homogenate and tested for ZEBOV or MARV using real-time or nested RT-PCR assays as previously described $[12,13]$. High-fidelity RT-PCR reagents (Biological Diagnostic Supplies Limited, Dreghorn, United Kingdom) were used for all conventional RT-PCR assays. ZEBOV nucleotide sequences [Genbank:DQ205409-DQ205415] and MARV nucleotide sequences [Genbank:EU068108EU068113] of some bats tested in this study have previously been described $[11,13]$.

\section{Statistical Analysis}

Access software (Microsoft 2007) was used for data analysis. Seroprevalence rates were compared with the Chisquare test and Fisher's test, implemented with Epi Info software (6.04, Epiconcept, France). Statistical significance was assumed at $\mathrm{p}<0.05$.

\section{Results}

\section{Bat collections}

We collected 2147 bats belonging to at least nine species (Figure 1): 1235 specimens (58\%) in Ogooué-Ivindo/ Mbomo (8 species), 440 (20\%) in Haut-Ogooué (8 species), 272 (13\%) in Moyen Ogooué (7 species) and 200 (9\%) in Nyanga (7 species). Most bats belonged to the species Epomops franqueti and Myonycteris torquata in
Ogooué-Ivindo, E. franqueti in Haut-Ogooué, Roussetus aegyptiacus in Moyen-Ogooué and R. aegyptiacus and $M$. torquata in Nyanga. Bats of six species were present at all the sites, namely E. franqueti (8-64\% of captured bats of this species by site), Hypsignathus monstrosus (1-8\%), Megaloglossus woermanni (1-10\%), Micropteropus pusillus (1$13 \%), R$. aegyptiacus (1-66\%) and M. torquata (1-36\%) (Figure 1). Twenty-four members of the Microchiroptera were captured but they were not all identified to the species level, owing to a lack of expertise in the field and because voucher specimens were not preserved.

\section{Prevalence of ZEBOV- and MARV-specific IgG}

Overall seroprevalence

Among the bats captured in Gabon and RC, 2147 were tested for ZEBOV-specific IgG and 1876 (87\%) for MARVspecific IgG. The respective prevalence rates were $4 \%$ ( $n=$ $95)$ and $1 \%(n=25)$ (Table 1$)$. Sera from 15 of the 95 ZEBOV-positive bats had OD values $>0.31$ when diluted 1:400, and one when diluted 1:1600 (Figure 3A). Sera from four of the 25 MARV-positive bats had OD values > 0.31 when diluted 1:400 (Figure 3B).

\section{Prevalence by species}

ZEBOV-specific IgG was found in 4 (2\%) of 197 Micropteropus pusillus, 19 (3\%) of 573 Myonycteris torquata, 36 (4\%) of 805 Epomops franqueti, 9 (7\%) of 125 Hypsignathus monstrosus, 24 (8\%) of 307 Rousettus aegyptiacus and 3 (12\%) of 24 Microchiroptera (Table 1). The prevalence of ZEBOV-specific IgG was significantly higher $(\mathrm{p}=0.005)$ in $R$. aegyptiacus specimens than in the bats of other species, with the exception of $H$. monstrosus ( $\mathrm{p}=0.82)$. The Microchiroptera group comprises several bat species and could not meaningfully compared with the bats of other species because of the small sample size. MARV-specific IgG was found in 21 (7\%) of 299 R. aegyptiacus, 1 (1\%) of $103 \mathrm{H}$. monstrosus, $1(0.6 \%)$ of $177 \mathrm{M}$. pusillus and $2(0.3 \%)$ of 679 E. franqueti (Table 1). Five R. aegyptiacus (3 from Haut-Ogooué, 1 each from Ogooué-Ivindo/RC and Moyen-Ogooué) were positive for both ZEBOV and MARV.

\section{Prevalence by site and year}

There were no significant differences $(p=0.7)$ in the prevalence of ZEBOV-specific IgG across the sites: 23 of 440 bats (5\%) in Haut-Ogooué, 12 of 272 (4\%) in Moyen Ogooué, 10 of 200 (5\%) in Nyanga and 50 of 1235 (4\%) in Ogooué-Ivindo/Mbomo (Figure 1). There was a temporal variation in the ZEBOV-specific IgG prevalence rates: in Mbomo (a region of documented Ebola outbreaks) the ZEBOV-specific IgG prevalence rate was 8 of $168(5 \%)$ in 2003, 26 of $314(8 \%)$ in 2005 and 10 of 494 (2\%) in 2006. In Haut-Ogooué the ZEBOV-specific IgG prevalence rate was 10 of $154(7 \%)$ in 2005, 3 of $130(2 \%)$ in 2006 and 3 of $73(4 \%)$ in 2008 (Table 2). 


\section{Ebola}

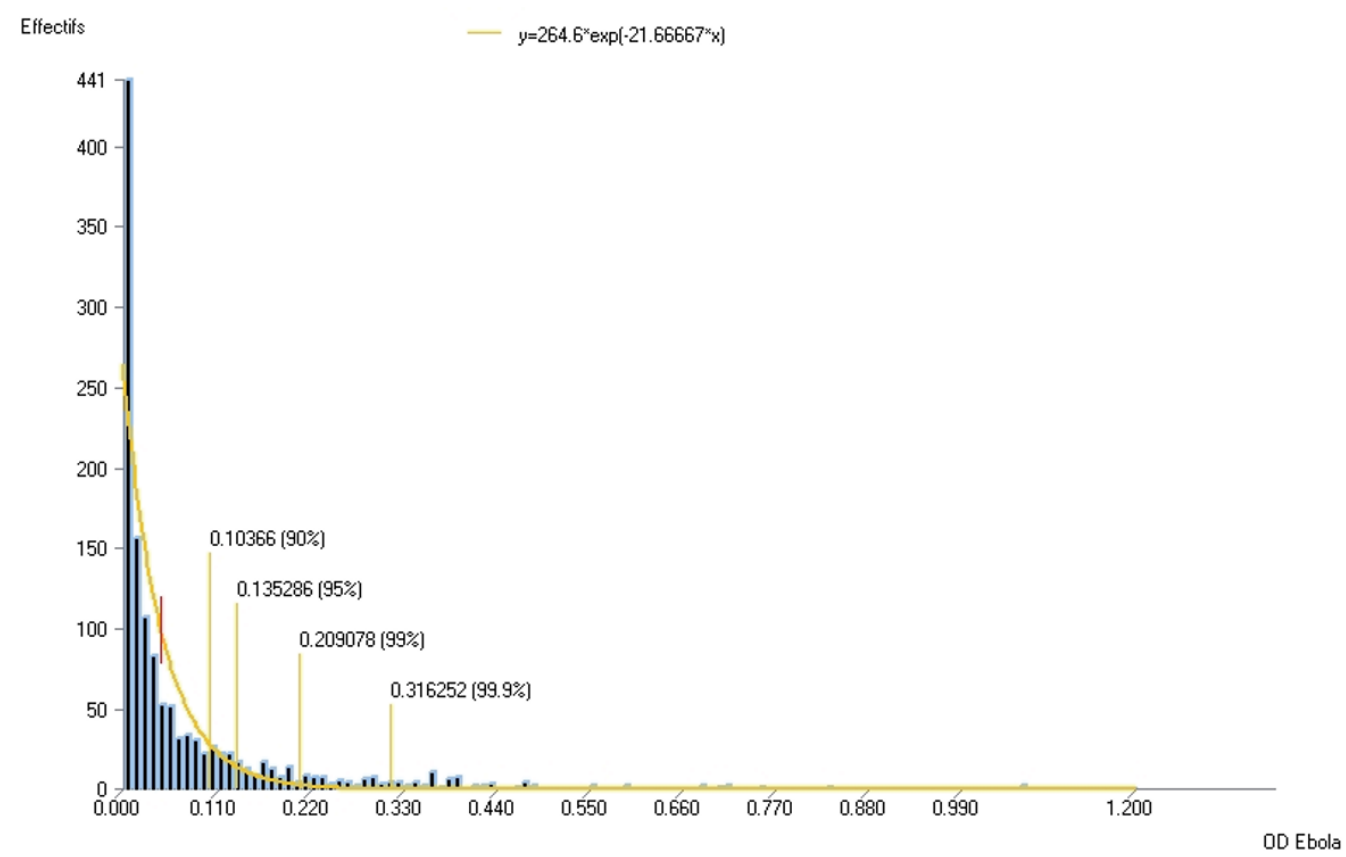

\section{Marburg}

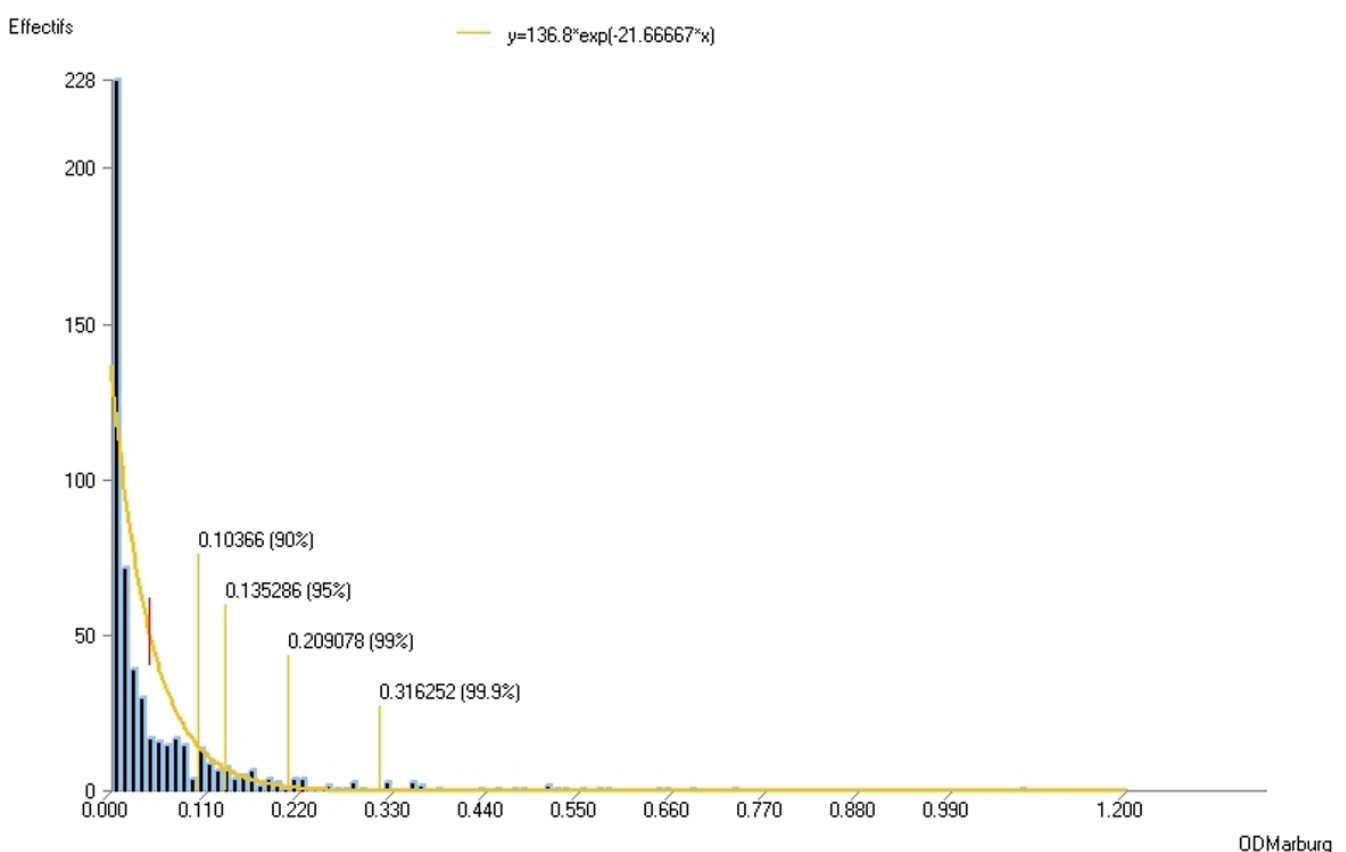

\section{Figure 2}

Determination of ZEBOV- and MARV-specific IgG prevalence cutoffs. The cut-off values were calculated assuming a negative exponential distribution of positive optical densities (OD). With a $0.00 \mathrm{I}$ risk of error, the cut-off was $0.3 \mathrm{I}$ for both Ebola and Marburg. 
Table I: Marburgvirus (MARV) and Zaire ebolavirus (ZEBOV) serological status (IgG) of bats captured between 2003 and 2008 in Gabon and Republic of Congo.

\begin{tabular}{lcc}
\hline & MARV & ZEBOV \\
\cline { 2 - 3 } Casinycteris argynnis & $0 / 18$ & $0 / 18$ \\
Eidolon helvum & $0 / 47$ & $0 / 49$ \\
Epomops franqueti & $2 / 679(0.3)$ & $36 / 805(4)$ \\
Hypsignathus monstrosus & $1 / 103(1)$ & $9 / 125(7)$ \\
Megaloglossus woermanni & $0 / 39$ & $0 / 49$ \\
Microchiroptera* & $0 / 20$ & $3 / 24(12)$ \\
Micropteropus pusillus & $1 / 177(0.6)$ & $4 / 197(2)$ \\
Rousettus aegyptiacus & $21 / 299(7)$ & $24 / 307(8)$ \\
Myonycteris torquata & $0 / 493$ & $19 / 573(3)$ \\
Total & $25 / 1876(1)$ & $95 / 2147(4)$ \\
\hline
\end{tabular}

* including Mops condylurus and Hipposideros gigas

Data are numbers of lgG-positive bats versus numbers of captured bats, and percentages

There were no significant differences $(\mathrm{p}=0.9)$ in the MARV-specific IgG prevalence rates between MoyenOgooué (5 of $264,1.9 \%$ ), Haut-Ogooué (10 of 435 , $2.2 \%$ ) and Nyanga ( 3 of $180,1.7 \%$ ) but the MARV-specific IgG prevalence rates were higher $(\mathrm{p}=0.003)$ in these regions than in Ogooué-Ivindo, where only three $(0.3 \%)$ of 997 bats were positive (Figure 1).

Eighty-seven specimens of $R$. aegyptiacus were captured in caves (in Nyanga and Haut-Ogooué) and 220 were captured elsewhere (in Nyanga, Haut-Ogooué and Moyen Ogooué) (Table 3). MARV-specific IgG was detected in 12 $(14 \%)$ of 84 cave-captured bats and in $9(4 \%)$ of 215 bats captured elsewhere $(\mathrm{p}=0.002)$. ZEBOV-specific IgG was detected in $8(9 \%)$ of $87 R$. aegyptiacus bats captured in caves and in $16(7 \%)$ of 220 members of this species sampled elsewhere $(\mathrm{p}=0.6)$ (Table 3$)$.

\section{Demographic parameters}

No significant difference was found in the prevalence of ZEBOV-specific IgG between males (44 of 922, 4\%) and females ( 51 of $1140,4 \%)(\mathrm{p}=0.7)$ or between adults $(79$ of $1653,5 \%)$ and juveniles (16 of 409, 4\%) $(\mathrm{p}=0.5)$ (Tables 4 and 5). However, the ZEBOV seroprevalence rate was higher $(\mathrm{p}=0.004)$ in pregnant females $(21(8 \%)$ of 268) than in non-pregnant females (22 (3\%) of 648). The prevalence of MARV-specific IgG was not different between males (13 of $822,2 \%$ ) and females (11 of 992 , $1 \%)(p=0.4)$, between adults ( 19 of $1444,1 \%)$ and juveniles $(5$ of $373,1 \%)(p=0.9)$ or between pregnant females ( 3 of 209, 1.9\%) and non pregnant females ( 4 of $577,1 \%)(\mathrm{p}=0.3)$ (Tables 4 and 6$)$.

\section{Virus genetic analysis}

ZEBOV nucleotide sequences were found in $13(5 \%)$ of 279 bats sampled in 2003 in RC [11], and MARV nucleotide sequences were found in $4(1 \%)$ of 283 bats sampled in 2005 in Gabon [13]. None of the other samples
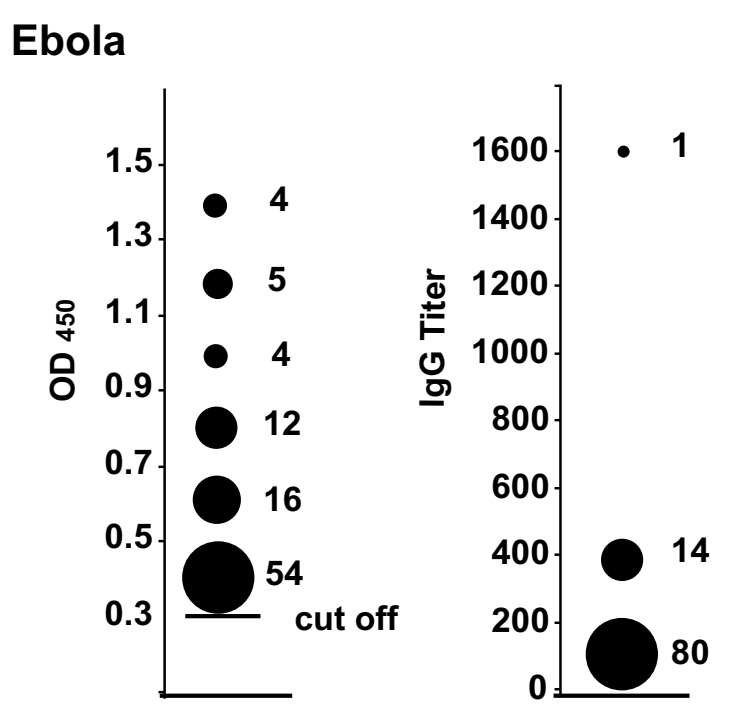

\section{Marburg}
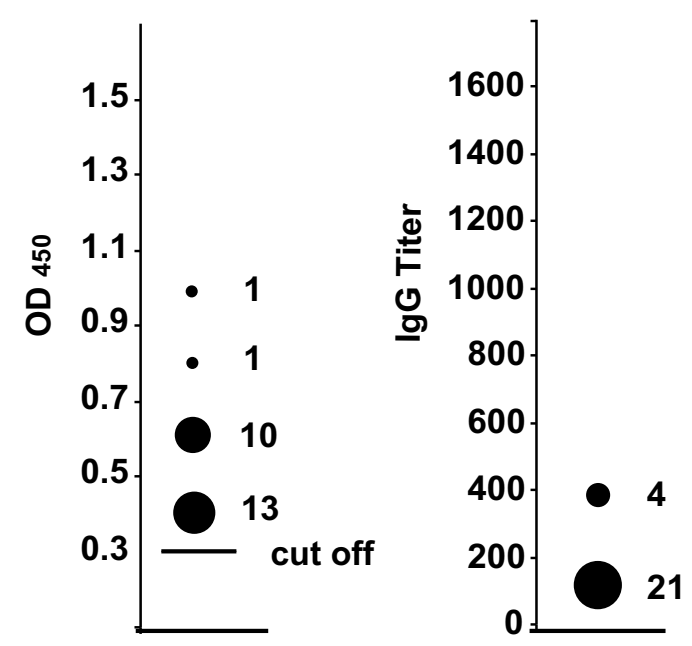

Figure 3

Corrected ODs and antibody titers in ZEBOV - and MARV-positive bats. The left side of the figure shows corrected OD values of I:I00-diluted bat sera with values higher than the 0.31 cutoff (solid horizontal bar). The numbers of sera used to calculate the values are shown to the right of the corresponding symbol. The right side of the figure shows IgG titers of sera with corrected OD values above $0.3 \mathrm{I}$. The numbers of sera used to calculate the values are shown to the right of the corresponding symbol.

analyzed was PCR-positive, including those from bats with ZEBOV- or MARV-specific IgG.

\section{Discussion}

The aim of this study was to elucidate the reported presence of ZEBOV- and MARV-specific antibodies in bats in Gabon. We had previously detected ZEBOV-specific IgG 
Table 2: Zaire ebolavirus (ZEBOV) serological status (IgG) of bats captured between 2003 and 2008 in Gabon and Republic of Congo, according to the collection site and period.

\begin{tabular}{|c|c|c|c|c|}
\hline Period of collection & E. franqueti & H. monstrosus & M. torquata & Total \\
\hline Mbomo 2003 & $4 / 100(4)$ & $2 / 18$ (II) & 2/50 (4) & $8 / 168(5)$ \\
\hline Mbomo 2005 & $10 / 132(8)$ & $5 / 38(13)$ & $11 / 144(8)$ & $26 / 3 \mid 4(8)$ \\
\hline Mbomo 2006 & $5 / 221(2)$ & $2 / 39(5)$ & $3 / 234(I)$ & $10 / 494(2)$ \\
\hline Total & $19 / 453(4)$ & $9 / 95(9)$ & $16 / 428(4)$ & $44 / 976(4)$ \\
\hline Haut-Ogooué 2005 & $8 / 109(7)$ & $0 / 1$ & $2 / 44(5)$ & $10 / 154(7)$ \\
\hline Haut-Ogooué 2006 & $3 / 113(3)$ & $0 / 1$ & $0 / 16$ & $3 / 130(2)$ \\
\hline Haut-Ogooué 2008 & $3 / 59(5)$ & $0 / 12$ & $0 / 2$ & $3 / 73(4)$ \\
\hline Total & $|4 / 28|(5)$ & $0 / 14$ & $2 / 62(3)$ & $16 / 357(4)$ \\
\hline
\end{tabular}

Data are numbers of ZEBOV IgG-positive bats versus numbers of captured bats, and (row) percentages. Only data for the three bat species suspected of being EBOV reservoirs are reported.

(in 16 of 192 bats) and ZEBOV RNA (in 13 of 279 bats) in E. franqueti, H. monstrosus and M. torquata specimens captured in the Ebola outbreak zone of northern Gabon and Congo between 2001 and 2003, and deduced that these three bat species might represent a ZEBOV reservoir [11]. Phylogenetic analyses of the seven sequences obtained from the 13 ZEBOV RNA positive bats showed very few differences relative to known human sequences (approximately 1\% of nucleotide positions in the L gene), confirming that Ebola virus detected in bats corresponded to the ZEBOV species. We subsequently detected MARVspecific IgG (in 29 of 242 bats) and MARV RNA (in 4 of 283 bats) in specimens of $R$. aegyptiacus captured in southern and southwestern Gabon in 2005 and deduced that this species might represent a MARV reservoir. Phylogenetic analysis of the MARV sequences obtained from the bats, in the N and VP 35 genes, showed approximately 5\% nucleotide divergence relative to the other western African Marburg virus lineage, Angola, a difference far smaller than the $15 \%$ diversity previously observed among East African Marburg virus isolates. This pointed to the existence of a geographic cluster including Angolan human MARV strains and Gabonese bat strains [13]. No blood samples were available from ZEBOV PCR-positive bats identified during our previous studies. However, all MARV PCR-positive bats were also positive for anti-MARV $\operatorname{IgG}[13]$.

Table 3: Marburgvirus (MARV) and zaire ebolavirus (ZEBOV) serological status (IgG) of bats of Rousettus aegyptiacus species in Gabon.

\begin{tabular}{lcc}
\hline & MARV & ZEBOV \\
\cline { 2 - 3 } Cave & $12 / 84(14)$ & $8 / 87(9)$ \\
No cave & $9 / 215(4)$ & $16 / 220(7)$ \\
Total & $21 / 299(7)$ & $24 / 307(8)$
\end{tabular}

Data are numbers of anti-ZEBOV IgG-positive bats versus numbers of captured bats, and (row) percentages.
Here we report the results of a large serological survey of ZEBOV and MARV in bats living in three regions of Gabon and northwest RC. In addition to the bats studied before, we examined additional specimens for ZEBOV infection ( 238 for IgG, 1468 for RNA) and for MARV (1438 for both IgG and RNA). We detected both ZEBOV-specific IgG (4\%) and MARV-specific IgG (1\%) in all four regions, confirming that the two viruses cocirculate in Gabon.

This is the first study to provide evidence of simultaneous ZEBOV and MARV circulation in bat populations in the same country. Several human ZEBOV outbreaks have occurred in Gabon, but no MARV outbreaks have been reported.

Interestingly, of the nine species reported here, specimens of six species (E. franqueti, $H$. monstrosus, M. torquata, $M$. pusillus, R. aegyptiacus and Mops condylurus) had ZEBOVspecific IgG and specimens of four species (R. aegyptiacus, H. monstrosus, E. franqueti, and M. pusillus) had MARV-specific IgG (Table 1). ZEBOV seroprevalence rates were high in four species, while a high MARV seroprevalence rate was found in only one species ( $R$. aegyptiacus). Together with the elevated antibody titers (at least 1:400) found in

Table 4: Demographic and ecological parametersassociated with marburgvirus (MARV) and zaire ebolavirus (ZEBOV) serological status (IgG) of bats captured between 2003 and 2008 in Gabon and Republic of Congo.

\begin{tabular}{lcc}
\hline & ZEBOV & MARV \\
\cline { 2 - 3 } Male/female & No & No \\
Pregnant female/non pregnant & Yes & No \\
Adult/juvenile & No & No \\
Cave/elsewhere & No & Yes \\
\hline
\end{tabular}

'Yes' signifies statistical significance $(p<0.05)$ and 'No' no statistical significance. Seroprevalence rates were compared with the Chisquare test implemented in Epi Info software (6.04, Epiconcept, France). 
Table 5: Zaire ebolavirus (ZEBOV) serological status (IgG) of bats captured between 2003 and 2008 in Gabon and Republic of Congo, according to demographic parameters.

\begin{tabular}{|c|c|c|c|c|c|c|c|c|c|}
\hline & \multicolumn{3}{|c|}{ Male } & \multicolumn{4}{|c|}{ Female } & \multirow[b]{2}{*}{ ND } & \multirow[b]{2}{*}{ Total } \\
\hline & Adult & Juvenile & Total & Adult Non pregnant & Adult pregnant & Juvenile & Total & & \\
\hline C. argynnis & $0 / 4$ & $0 / 0$ & $0 / 4$ & $0 / 9$ & $0 / 4$ & $0 / 1$ & $0 / 14$ & $0 / 0$ & $0 / 18$ \\
\hline E. helvum & $0 / 11$ & $0 / 7$ & $0 / 18$ & $0 / 17$ & $0 / 0$ & $0 / 11$ & $0 / 28$ & $0 / 3$ & $0 / 49$ \\
\hline E. franqueti & $14 / 269(5)$ & $2 / 87(2)$ & $16 / 356(4)$ & $9 / 243(4)$ & $9 / 132(7)$ & $2 / 36(6)$ & $20 / 4 I I(5)$ & $0 / 8$ & $36 / 805(4)$ \\
\hline H. monstrosus & I/34(3) & $2 / 19(11)$ & $3 / 53(6)$ & $1 / 18(6)$ & $3 / 18(17)$ & $2 / 33(6)$ & $6 / 69(9)$ & $0 / 3$ & $9 / 125(7)$ \\
\hline M. woermanni & $0 / 19$ & $0 / 0$ & $0 / 19$ & $0 / 21$ & $0 / 5$ & $0 / 4$ & $0 / 30$ & $0 / 0$ & $0 / 49$ \\
\hline Microchiroptera & $1 / 9(11)$ & $0 / 2$ & $I / I I(9)$ & $2 / 5(40)$ & $0 / 0$ & $0 / 0$ & $2 / 5(40)$ & $0 / 8$ & $3 / 24(12)$ \\
\hline M. pusillus & $0 / 76$ & $0 / 3$ & $0 / 79$ & $1 / 36(3)$ & $3 / 53(6)$ & $0 / 22$ & $4 / I I I(4)$ & $0 / 7$ & $4 / 197(2)$ \\
\hline R. aegyptiacus & $11 / 88(13)$ & $4 / 35(12)$ & $15 / 123(12)$ & $3 / 90(3)$ & $3 / 13(23)$ & $3 / 78(4)$ & $9 / 181(5)$ & $0 / 3$ & $24 / 307(8)$ \\
\hline M. torquata & $9 / 227(4)$ & $0 / 32$ & $9 / 259(3)$ & 6/209(3) & $3 / 43(7)$ & $1 / 39(3)$ & 10/291(3) & $0 / 23$ & 19/573(3) \\
\hline Total & $36 / 737(5)$ & $8 / 185(4)$ & $44 / 922(4)$ & $22 / 648(3)$ & $21 / 268(8)$ & $8 / 224(4)$ & $51 / 1140(4)$ & $0 / 85$ & $95 / 2147(4)$ \\
\hline
\end{tabular}

Data are numbers of ZEBOV-specific IgG-positive bats versus numbers of captured bats, and percentages.

these bats, these finding indicate that these bats have frequent contacts with ZEBOV and MARV.

In an earlier study in DRC, members of three bat species were found to be seropositive for MARV ( $R$. aegyptiacus and two members of the Microchiroptera group: Rhinolophus eloquens and Miniopterus inflatus) [14], possibly because they roosted in close proximity at the capture sites. In Gabon, no specimens belonging to these last two species were captured during our field studies. Surprisingly, ZEBOV-specific IgG was also frequent in R. aegyptiacus $(8 \%)$. No specimens of this species were sampled between 2001 and 2003 in northern Gabon/Congo $[11,15]$. ZEBOV seropositivity in R. aegyptiacus bats likely reflects true viral infection, although passive antigenic stimulation cannot be ruled out. In Gabon, Megachiroptera members, including $R$. aegyptiacus, E. franqueti, $M$. torquata and $H$. monstrosus bats, consume the same fruits (especially Ficus spp). Bats of different species tend to visit the same trees, and interspecies virus transmission (or simple antigenic stimulation) could occur via infected saliva deposited on fruits. Hendra virus and Nipah virus transmission has been suggested to occur in this way between bats belonging to Asian Pteropus species [16].

Among IgG-positive R. aegyptiacus specimens, 21 (42\%) were positive for MARV, 24 (48\%) were positive for ZEBOV, and $6(10 \%)$ were positive for both viruses. Although dual infection and/or antigenic stimulation could explain the concomitant presence of ZEBOV- and MARV-specific IgG in individual bats, serological crossreactions between Ebola and Marburg antigens cannot be ruled out.

The ZEBOV seroprevalence rates were very similar (4\% to $5 \%$ ) in the four sampling regions (Figure 1). By contrast, the MARV seroprevalence rate was higher (about 2\%) in the three regions where R. aegyptiacus was abundantly captured. Although some groups of $R$. aegyptiacus may occasionally roost outside in the trees, the bats of this species usually roost in natural or man-made cavities (caves, mines, tombs, etc.) and search for food at night. The daily

Table 6: Marburgvirus (MARV) serological status (IgG) of bats captured between 2003 and 2008 in Gabon and Republic of Congo, according to demographic parameters.

\begin{tabular}{|c|c|c|c|c|c|c|c|c|c|}
\hline & \multicolumn{3}{|c|}{ Male } & \multicolumn{4}{|c|}{ Female } & \multirow[b]{2}{*}{ ND } & \multirow[b]{2}{*}{ Total } \\
\hline & Adult & Juvenile & Total & Adult Non pregnant & Adult pregnant & Juvenile & Total & & \\
\hline C. argynnis & $0 / 4$ & $0 / 0$ & $0 / 4$ & $0 / 9$ & $0 / 4$ & $0 / 1$ & $0 / 14$ & $0 / 0$ & $0 / 18$ \\
\hline E. helvum & $0 / 11$ & $0 / 7$ & $0 / 18$ & $0 / 16$ & $0 / 0$ & $0 / 11$ & $0 / 27$ & $0 / 2$ & $0 / 47$ \\
\hline E. franqueti & $\mathrm{I} / 234(0.3)$ & $0 / 80$ & $1 / 3 \mid 4(0.3)$ & $|/ 2| 4(0.5)$ & $0 / 88$ & $0 / 31$ & $1 / 333(0.3)$ & $0 / 32$ & $2 / 679(0.3)$ \\
\hline H. monstrosus & $0 / 32$ & $0 / 15$ & $0 / 47$ & $0 / 14$ & $0 / 13$ & $1 / 28(4)$ & I/55(2) & $0 / 1$ & $1 / 103(1)$ \\
\hline M. woermanni & $0 / 16$ & $0 / 0$ & $0 / 16$ & $0 / 15$ & $0 / 5$ & $0 / 3$ & $0 / 23$ & $0 / 0$ & $0 / 39$ \\
\hline Microchiroptera & $0 / 8$ & $0 / 0$ & $0 / 8$ & $0 / 5$ & $0 / 0$ & $0 / 0$ & $0 / 5$ & $0 / 7$ & $0 / 20$ \\
\hline M. pusillus & $1 / 69(1)$ & $0 / 3$ & I/72(I) & $0 / 32$ & $0 / 48$ & $0 / 21$ & $0 / 101$ & $0 / 4$ & $\mathrm{I} / \mathrm{I77}(0.6)$ \\
\hline R. aegyptiacus & $10 / 87(11)$ & $1 / 33(3)$ & $11 / 120(1)$ & $3 / 87(3)$ & $4 / 13(30)$ & $3 / 76(4)$ & $10 / 176(4)$ & $0 / 3$ & $21 / 299(7)$ \\
\hline M. torquata & $0 / 197$ & $0 / 26$ & $0 / 233$ & $0 / 185$ & $0 / 38$ & $0 / 35$ & $0 / 258$ & $0 / 15$ & $0 / 494$ \\
\hline Total & $12 / 658(2)$ & $1 / 164(0.6)$ & $13 / 822(2)$ & $4 / 577(I)$ & $3 / 209(1)$ & $4 / 206(2)$ & $11 / 992(1)$ & $0 / 62$ & $25 / 1876(1)$ \\
\hline
\end{tabular}

Data are numbers of ZEBOV-specific lgG-positive bats versus numbers of captured bats, and percentages. 
flight distances are unknown. Among the 299 R. aegyptiacus specimens sampled in Gabon, the MARV-specific IgG prevalence rate was significantly higher in the animals captured in caves $(14 \%)$ than in those captured elsewhere (4\%) (Table 3). This difference could be due to a higher circulation of MARV between the bats living in the caves. Similarly, a high MARV-specific IgG prevalence rate (about 20\%) has been reported in DRC in a cave-captured population of R. aegyptiacus [14]. Together, these findings point to the existence of two different ecological biotopes for Marburg and Ebola viruses: one linked to bats roosting in caves (Rousettus or Microchiroptera species) and the other linked to bats roosting in trees in the open forest. The ecology of $H$. monstrosus depends on water supplies, and these bats are known to migrate locally, especially along rivers [17]. Thus, the geographic distribution of ZEBOV in its reservoir species may be very broad, encompassing the forested areas of Africa, especially close to rivers, as previously reported [6,7]. In contrast, the distribution of Marburg virus would be determined by the ecology (horizontal and vertical distribution) of $R$. aegyptiacus bats and the presence of caves. Our serological results strongly suggest that ZEBOV and MARV overlap in all four regions we sampled in Gabon and RC.

The prevalence of ZEBOV-specific IgG fell from about 5\% in 2003 and $8 \%$ in 2005 in Mbomo and 7\% in HautOgooué (during ZEBOV outbreaks) to $2 \%$ at both sites in 2006 (one year after the last outbreak), before rising again to $4 \%$ in Haut-Ogooué in 2008 (Table 2). Similar seasonal variations in the IgG prevalence of other viral pathogens (European Bat Lyssavirus-1, Hendra virus, etc.) have also been described in bats $[18,19]$. These fluctuations could be due to factors such as changes in bat numbers (due to mortality, migration, influx of young animals, etc.), immunological parameters (antibody persistence) and environmental changes (fructification, etc.). Long-term studies of seasonal variations in the ZEBOVspecific IgG prevalence in bat populations might help to understand the dynamics of human Ebola outbreaks.

The higher prevalence of ZEBOV-specific IgG in pregnant females $(8 \%)$ than in non pregnant females $(3 \%)$ (Table 5) suggests that EBOV transmission between males and females could occur by contact with infected saliva (bites, licking) or by mating. Pregnant female Pteropus scapulatus bats in Australia were recently shown to be more susceptible than their non pregnant counterparts to Hendra virus infection, and it has been suggested that Hendra virus transmission may occur by contact with the infected placenta [19].

\section{Conclusion}

We confirm the cocirculation of Ebola and Marburg viruses in Gabon. To date, Gabon is the only country where bat reservoirs of both viruses have also been identified. Of all the bats sampled in significant numbers in this survey, only specimens of Rousettus aegyptiacus were found to harbor both MARV- and ZEBOV-specific IgG, suggesting that this species may be a natural host of both viruses. The presence of MARV in Gabon, where no human Marburg hemorrhagic fever outbreaks have been reported, represents a potential and previously unrecognized threat to humans. As the MARV seroprevalence was significantly higher in $R$. aegyptiacus bats captured inside caves than elsewhere, caves and their surroundings should be considered as high-risk areas.

\section{Competing interests}

The authors declare that they have no competing interests.

\section{Authors' contributions}

Designed and performed the experiments: XP and EL. Analyzed the data: XP, EL, MS, PR, JT, SN and JPG. Statistical analysis MS. Contributed reagents/materials: PR, SN and JT. Wrote the paper: XP and EL. All authors read and approved the final version of the manuscript.

\section{Acknowledgements}

CIRMF is supported by the Government of Gabon, Total-Fina-Elf Gabon, and Ministère de la Coopération Française. This work was also supported by a Fonds de Solidarité Prioritaire grant from Ministère des Affaires Etrangères de la France (FSP $n^{\circ}$ 2002005700).

We thank all the persons involved in sample collection, and especially André Délicat, Patrick Yangari and Philippe Yaba.

\section{References}

I. Feldmann H, Kiley MP: Classification, structure, and replication of filoviruses. Curr Top Microbiol Immunol 1999, 235:I-2I.

2. Towner JS, Sealy TK, Khristova ML, Albarino CG, Conlan S, Reeder SA, Quan PL, Lipkin WI, Downing R, Tappero JW, et al.: Newly discovered ebola virus associated with hemorrhagic Fever outbreak in Uganda. PLoS Pathog 2008, 4(I I): e I0002 I 2.

3. Pourrut $X$, Kumulungui B, Wittmann T, Moussavou G, Délicat A, Yaba P, Nkoghé D, Gonzalez JP, Leroy EM: The natural History of Ebola virus in Africa. Microbes Infect 2005, 7:1005-1014.

4. Wittmann TJ, Biek R, Hassanin A, Rouquet P, Reed P, Yaba P, Pourrut $X$, Real LA, Gonzalez JP, Leroy EM: Isolates of Zaire ebolavirus from wild apes reveal genetic lineage and recombinants. Proc Natl Acad Sci USA 2007, I04(43): I7123-I7I 27.

5. Martini GA: Marburg agent disease in man. Trans $R$ Soc Trop Med Hyg 1969, 63:295-302.

6. Peterson AT, Bauer JT, Mills JN: Ecologic and geographic distribution of filovirus disease. Emerg Infect Dis 2004, I 0:40-47.

7. Peterson AT, Lash RR, Carroll DS, Johnson KM: Geographic potential for outbreaks of Marburg hemorrhagic fever. $\mathrm{Am} J$ Trop Med Hyg 2006, 75(I):9-I5.

8. Leroy EM, Rouquet $P$, Formenty $P$, Souquière $S$, Kilbourne A, Froment J-M, Bermejo M, Smit S, Karesh W, Swanepeol R, et al.: Multiple Ebola virus transmission events and rapid decline of central african wildlife. Science 2004, 303:387-390.

9. Swanepoel R, Leman PA, Burt FJ: Experimental inoculation of plants and animals with Ebola virus. Emerg Infect Dis 1996, 2:321-325.

10. Peterson AT, Carroll DS, Mills JN, Johnson AM: Potential mammalian filovirus reservoirs. Emerg Infect Dis 2004, I 0:2073-208I.

II. Leroy EM, Kumulungui B, Pourrut X, Rouquet $P$, Hassanin A, Yaba $P$, Delicat A, Paweska JT, Gonzalez JP, Swanepoel R: Fruit bats as reservoirs of Ebola virus. Nature 2005, 438(7068):575-576. 
12. Pourrut X, Delicat A, Rollin PE, Ksiazek TG, Gonzalez JP, Leroy EM: Spatial and temporal patterns of Zaire ebolavirus antibody prevalence in the possible reservoir bat species. J Infect Dis 2007, 196(Suppl 2):SI76-|83.

13. Towner JS, Pourrut X, Albarino CG, Nkogue CN, Bird BH, Grard G, Ksiazek TG, Gonzalez JP, Nichol ST, Leroy EM: Marburg virus infection detected in a common African bat. PLOS ONE 2007, 2(I):e764.

14. Swanepoel R, Smit SB, Rollin PE, Formenty P, Leman PA, Kemp A, Burt F], Grobbelaar AA, Croft J, Bausch DG, et al:: Studies of reservoir hosts for Marburg virus. Emerg Infect Dis 2007, I3(12):|847-185|.

15. Gonzalez JP, Pourrut X, Leroy E: Ebolavirus and other filoviruses. Curr Top Microbiol Immunol 2007, 3 I 5:363-387.

16. Williamson MM, Hooper PT, Selleck PW, Gleeson LJ, Daniels PW, Westbury HA, Murray PK: Transmission studies of Hendra virus (equine morbillivirus) in fruit bats, horses and cats. Aust Vet J 1998, 76(12):813-818.

17. Kingdom J: East african mammals. University of Chigaco Press Edition; Chigaco; 1984.

18. Amengual B, Bourhy H, Lopez-Roig M, Serra-Cobo J: Temporal dynamics of European bat Lyssavirus type $I$ and survival of Myotis myotis bats in natural colonies. PLOS ONE 2007, 2(6): 5566 .

19. Plowright RK, Field HE, Smith C, Divljan A, Palmer C, Tabor G, Daszak P, Foley JE: Reproduction and nutritional stress are risk factors for Hendra virus infection in little red flying foxes (Pteropus scapulatus). Proc Biol Sci 2008, 275(1636):86 I-869.

\section{Pre-publication history}

The pre-publication history for this paper can be accessed here:

http://www.biomedcentral.com/1471-2334/9/159/pre pub

Publish with Bio Med Central and every scientist can read your work free of charge

"BioMed Central will be the most significant development for disseminating the results of biomedical research in our lifetime. "

Sir Paul Nurse, Cancer Research UK

Your research papers will be:

- available free of charge to the entire biomedical community

- peer reviewed and published immediately upon acceptance

- cited in PubMed and archived on PubMed Central

- yours - you keep the copyright

Submit your manuscript here:

http://www.biomedcentral.com/info/publishing_adv.asp 